\title{
Forward Stimulated Brillouin Scattering in Free-Standing Waveguides on a Silicon Photonics Platform
}

\author{
Khannan Rajendran ${ }^{1,2}$, Awanish Pandey ${ }^{1,2}$ Alain Yuji Takabayashi ${ }^{3}$, Umar \\ Khan $^{1,2}$, Wim Bogaerts ${ }^{1,2}$, Niels Quack ${ }^{3}$, Dries Van Thourhout ${ }^{1,2}$ \\ (1) Photonics Research Group, Gent University-imec, Gent, Belgium \\ (2) Center for Nano- and Biophotonics, Gent University-imec, Gent, Belgium \\ (3) Ecole Polytechnique Fédérale de Lausanne (EPFL), 1015 Lausanne, Switzerland \\ Khannan.Rajendran@Ugent.be
}

\begin{abstract}
Stimulated Brillouin Scattering (SBS) is demonstrated on a standard active silicon photonics platform, the forward SBS gain and opto-mechanical coupling rates are calculated for two different rib waveguide geometries. (C) 2021 The Author(s)
\end{abstract}

\section{Introduction}

Stimulated Brillouin Scattering (SBS) is the nonlinear interaction between photons and acoustic phonons, with applications in microwave photonics, and signal processing [1]. Here, we demonstrate SBS in passive silicon waveguides on IMEC's active silicon-on-insulator photonics platform iSiPP50G, which have undergone custom post-processing to provide suspended waveguides [2]. These passive suspended waveguides are tightly integrated next to high performance active components of the platform, such as modulators, detectors, and phase shifters. We first present the design of the waveguide, then elaborate its fabrication procedure and finally report our preliminary results on the SBS investigation in these waveguides.

\section{Design and fabrication}

We consider waveguides with two different etch depths: single mode rib (FC - 70nm deep etch) and socket (SKT $150 \mathrm{~nm}$ deep etch) waveguides as shown in Fig. 1. Both waveguides are $2000 \mu \mathrm{m}$ long. Trenches of $5.5 \mu \mathrm{m} \times 1 \mu \mathrm{m}$ with a pitch of $6 \mu \mathrm{m}$ are patterned in the $220 \mathrm{~nm}$ thick silicon device layer at a distance of $1.45 \mu \mathrm{m}$ from the waveguide core to facilitate oxide cladding etching. The buried oxide cladding layer ( $2 \mu \mathrm{m}$ thick) has been removed by HF vapour etchant to suspend the waveguide. More details about the etch process can be found in [3].

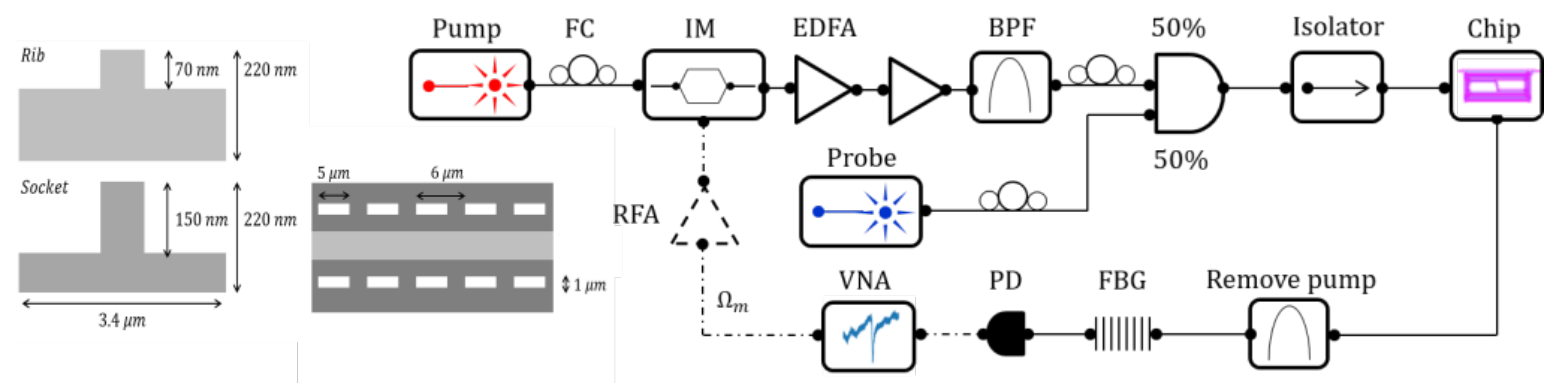

Fig. 1. Left (in grey) : Schematic of the rib (FC) and socket (SKT) waveguide cross section along with the trench profile. Right : Schematic of the cross phase modulation (XPM) experimental setup.

\section{Cross-phase modulation experiment}

We observe forward SBS in the rib waveguides by measuring the cross-phase modulation (XPM) imprinted on a weak probe by a strongly modulated pump [4] as shown in Fig. 1. A pre-amplified intensity modulated (IM) pump laser $(1540 \mathrm{~nm})$ with a continuous probe $(1550.28 \mathrm{~nm})$, are combined and injected into the chip via grating couplers (insertion loss of $7 \mathrm{~dB} /$ coupler). The pump excites a non-resonant Kerr and a narrow-band resonant Brillouin response in the suspended waveguides. The coherent interference of these two non-linearities results in a Fano signature, which is imprinted on the probe through XPM. At the output, the pump is removed using a band pass filter (BPF). The anti-Stokes side-band of the probe is rejected using a fiber Bragg grating (FBG) with a steep 

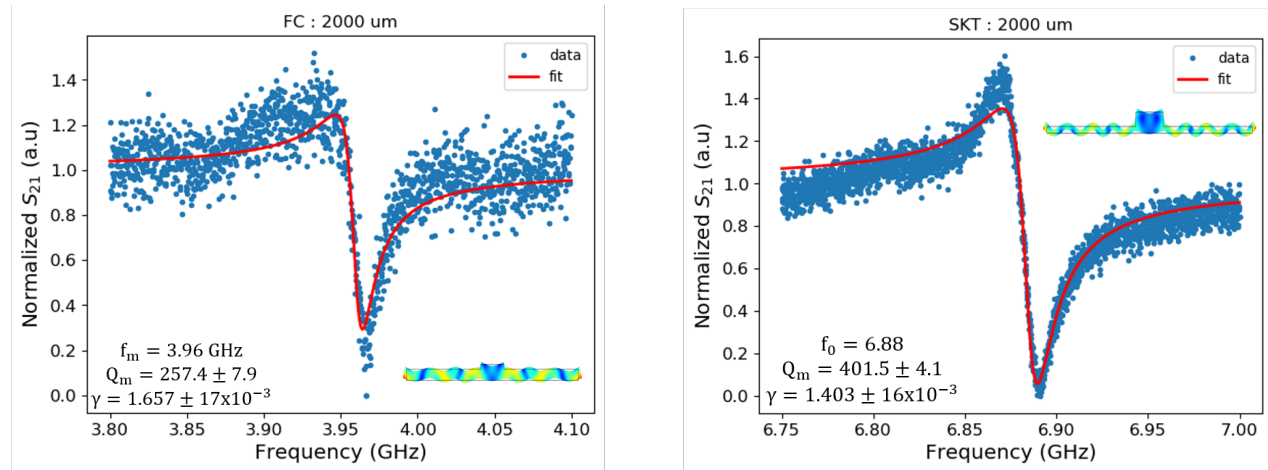

Fig. 2. Fano resonances for $2000 \mu m$ long spirals - Left: FC (deep) etch rib waveguides. Right: SKT (shallow) etch rib waveguides. Inset are the fit parameters [4] and the corresponding mechanical resonance mode, simulated using COMSOL.

roll-off (30 dB extinction over 2.5 GHz). The FBG converts the phase modulated probe into intensity modulation, to be detected by a photodetector (PD). The RF output from the PD, containing the Fano signature is observed using a VNA.

The background normalized asymmetric Fano response as shown in Fig. 2, can be fitted [4] to extract the resonant frequency $\left(\Omega_{m} / 2 \pi\right)$, mechanical Q-factor $\left(Q_{m}\right)$ and Brillouin non-linearity $\left(\gamma_{S B S}\right)$, as presented in Tab. 1 for the different waveguide shapes. In addition, we also calculate the equivalent optomechanical coupling rate $\left(g_{0}\right)[5]$ for the $2000 \mu m$ long waveguides.

Table 1. Key figures of merit extracted from XPM-fano resonances (in Fig. 2).

\begin{tabular}{|c||c|c|c|c||c|c|c|c|}
\hline \multicolumn{1}{|c||}{} & \multicolumn{4}{c||}{ FC } & \multicolumn{4}{c|}{ SKT } \\
\hline length & $f_{m}$ & $Q_{m}$ & $\gamma_{S B S}$ & $g_{0}$ & $f_{m}$ & $Q_{m}$ & $\gamma_{S B S}$ & $g_{0}$ \\
\hline$\mu m$ & $G H z$ & - & $\times 10^{3} / W / m$ & $M H z$ & $G H z$ & - & $\times 10^{3} / W / m$ & $M H z$ \\
\hline \hline 2000 & 3.96 & $257 \pm 7$ & $3.112 \pm 0.031$ & $0.230 \pm 0.003$ & 6.884 & $402 \pm 4$ & $2.636 \pm 0.003$ & $0.220 \pm 0.002$ \\
\hline
\end{tabular}

\section{Conclusions}

We show forward SBS in a full silicon photonics platform for a shallow and deep etch waveguide, using an crossphase modulation based detection scheme. We observe a Brillouin non-linearity $\gamma_{S B S}$ of $3112 \frac{1}{W \cdot m}$ and $2636 \frac{1}{W \cdot m}$ for a $2000 \mu \mathrm{m}$ FC and SKT spiral respectively.

\section{Acknowledgements}

This work was supported by the EU H2020 grant agreements OMT (No. 722923) and MORPHIC (No.780283), and the EU commission through grant FET proactive HOT (No. 732894).

\section{References}

1. B. J. Eggleton, C. G. Poulton, P. T. Rakich, M. J. Steel, and G. Bahl, "Brillouin integrated photonics," Nat. Photonics 13, 664-677 (2019).

2. W. Bogaerts, H. Sattari, P. Edinger, A. Y. Takabayashi, I. Zand, X. Wang, A. Ribeiro, M. Jezzini, C. Errando-Herranz, G. Talli et al., "Morphic: Programmable photonic circuits enabled by silicon photonic mems," in Silicon Photonics XV, , vol. 11285 (International Society for Optics and Photonics, 2020), p. 1128503.

3. N. Quack, H. Sattari, A. Y. Takabayashi, Y. Zhang, P. Verheyen, W. Bogaerts, P. Edinger, C. Errando-Herranz, and K. B. Gylfason, "Mems-enabled silicon photonic integrated devices and circuits," IEEE J. Quantum Electron. 56, 1-10 (2019).

4. R. Van Laer, B. Kuyken, D. Van Thourhout, and R. Baets, "Interaction between light and highly confined hypersound in a silicon photonic nanowire," Nat. Photonics 9, 199-203 (2015).

5. R. Van Laer, R. Baets, and D. Van Thourhout, "Unifying brillouin scattering and cavity optomechanics," Phys. Rev. A 93, 053828 (2016). 